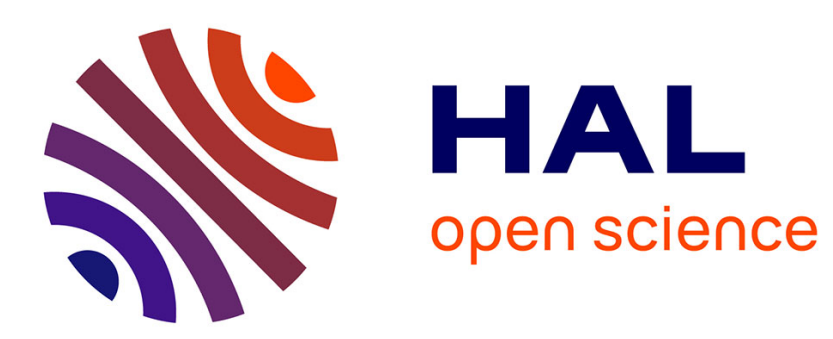

\title{
Reducing collision probability on a shared medium using a variational method
}

Michel Marot, Vincent Gauthier

\section{To cite this version:}

Michel Marot, Vincent Gauthier. Reducing collision probability on a shared medium using a variational method. MAMA 2010: 12th Workshop on MAthematical Performance Modeling and Analysis, Jun 2010, New York United States. hal-01354822

\section{HAL Id: hal-01354822 \\ https://hal.science/hal-01354822}

Submitted on 19 Aug 2016

HAL is a multi-disciplinary open access archive for the deposit and dissemination of scientific research documents, whether they are published or not. The documents may come from teaching and research institutions in France or abroad, or from public or private research centers.
L'archive ouverte pluridisciplinaire HAL, est destinée au dépôt et à la diffusion de documents scientifiques de niveau recherche, publiés ou non, émanant des établissements d'enseignement et de recherche français ou étrangers, des laboratoires publics ou privés. 


\title{
Reducing Collision Probability on a shared medium using a variational method
}

\author{
Michel Marot, Vincent Gauthier \\ Laboratoire SAMOVAR \\ Télécom SudParis, 9 rue C. Fourier, 91011 Evry CEDEX, France \\ \{michel.marot,vincent.gauthier\}@telecom-sudparis.eu
}

\begin{abstract}
We consider a network with $N$ nodes competing for access to the channel using un-slotted ALOHA. When a request is sent, each node may answer after a certain backoff time. Only the first answer is of importance, that is why we want to minimize the loss rate of the first message. We derive the optimal backoff probability distribution which minimizes the collision probability on the first message answering to a request. Unlike previous works, we extract the collision probability in continuous time domain. To this goal, we use a variational method. This problem had only be solved before in a slotted context (i.e. discrete time domain), but we want to be able to manage later situations where the nodes are not perfectly synchronized, which requires to know how to solve it in the continuous time context.
\end{abstract}

\section{Keywords}

Medium access, ALOHA, random access, first message, collision probability, variational calculus

\section{INTRODUCTION}

In wireless networks, especially in sensor networks, situations often occur where only the first reply to a request is important. It is typically the case with election protocols (cf. [1] for example, or [2], [3]). Usually, an ALOHA mechanism is used to transmit the answers, and nodes derive their backoff times (according to certain probability distribution) before transmitting the answer. In order to optimize the performance of such protocols, we tried to identify the best distribution which minimizes the collision probability of the first answer that collides with the replies of the other nodes.

Several interesting works have been published until now. In [4], authors address exactly this issue in a context where the time is slotted. But we want to relax this assumption on the slotted synchronization between the nodes. This may be difficult to implement in some situations and a method is needed to deal with un-slotted situations. In [6] and [7], the same problem in the same slotted context is addressed, but the authors noticed that the collision probability can be reduced if the probability that the nodes answer is less than 1. An optimal value is given. Moreover, the solution is explicit in [7] while it is only recursive in [6]. In [5], the authors address the same question without assuming any synchronization, but they do not derive analytically an exact solution. Moreover, there is a mistake in the formula (7) of [5] where it is implicitely assumed a uniform distribution for the loss rate, we give the correct formula below: formula (7).

In this work, we identify the optimal backoff distribution mechanism in a non-slotted environment. The problem is then to find an optimal continuous function minimizing an integral. Then, we use the classical tools of the variational calculus to derive the solution.

\section{MODELING ASSUMPTIONS AND FOR- MALIZATION OF THE PROBLEM}

We reuse the same formalization as in [5]. We consider a node having $N$ neighbors. It sends a query and each one of the other nodes sends a reply after a given backoff time. We are interested by the first answer. Collisions involving messages other than the first one is not considered to be a problem. Each node has a window of length $D$. We assume $t=0$ at the beginning of the backoff period which corresponds to the receipt instant of the query by all the $N$ nodes. Let $x_{1}, x_{2}, \cdots, x_{N}$ be the times when the expected answers of node $i \in[1 ; N]$ are sent. The duration of the messages is considered the same for all the messages and equal to $d$. Let $x_{\text {first }}$ denote the minimum of the $x_{i}$ : $x_{\text {first }}=\min _{i \in[1 ; N]} x_{i}$. The collision probability of the first message is denoted $P_{(D, N)}$ and is formally given by:

$$
\begin{aligned}
P_{(D, N)=} & P(\forall i \in[1 ; N] \\
& \left.x_{i}<x_{\text {first }}+d / x_{i} \neq x_{\text {first }}\right)
\end{aligned}
$$

Let $\forall x \in[0 ; D], y(x)$ be the density function of the backoff distribution.

3. CALCULATION OF $P_{(D, N)}$

Let us assume $N=2$. When the first message is emitted at $x_{\text {first }}$, the probability that it collides with the second answer 
is:

$$
p_{1}=\frac{\int_{x_{f i r s t}}^{x_{f i r s t}+d} y(x) d x}{\int_{x_{\text {first }}}^{D} y(x) d x}
$$

Then, with $\mathrm{N}$ independent nodes, this probability is, for all $x$ in $[0 ; D-d]$ :

$$
\begin{aligned}
p_{N-1} & =1-\left[1-\frac{\int_{x_{f i r s t}}^{x_{f i r s t}+d} y(x) d x}{\int_{x_{f i r s t}}^{D} y(x) d x}\right]^{N-1} \\
& =1-\left[\frac{\int_{x_{f i r s t}+d}^{D} y(x) d x}{\int_{x_{\text {first }}}^{D} y(x) d x}\right]^{N-1}
\end{aligned}
$$

When $x$ is in $[D-d ; D]$, the collision probability is equal to 1 . Thus, the mean loss rate for any $x_{\text {first }}$ is:

$$
\begin{aligned}
P_{(D, N)}= & \int_{0}^{D-d} 1-\left[\frac{\int_{x_{f i r s t}+d}^{D} y(x) d x}{\int_{x_{f i r s t}}^{D} y(x) d x}\right]^{N-1} y_{f i r s t}(x) d x \\
& +\int_{D-d}^{D} y_{f i r s t}(x) d x
\end{aligned}
$$

where $y_{\text {first }}(x)$ is the distribution of $x_{\text {first }}$. Let us determine $y_{\text {first }}$. The cumulative distribution function of $x_{f i r s t}$ is

$$
\begin{aligned}
P\left(x_{\text {first }}<x\right)= & P\left(\min _{i \in[1 ; N]} x_{i}<x\right) \\
& =1-P\left(\min _{i \in[1 ; N]} x_{i} \geq x\right) \\
& =1-\prod_{1}^{N}\left(x_{i} \geq x\right) \\
& =1-\left(\int_{x}^{D} y(u) d u\right)^{N}
\end{aligned}
$$

and thus, its density function is obtained by differentiation:

$$
\frac{d}{d x} P\left(x_{\text {first }}<x\right)=N y(x)\left(\int_{x}^{D} y(u) d u\right)^{N-1}
$$

The general formula for the mean collision probability is then:

$$
\begin{aligned}
P_{(D, N)}= & \int_{0}^{D-d}\left[1-\left(\frac{\int_{x_{f i r s t}+d}^{D} y(x) d x}{\int_{x_{f i r s t}}^{D} y(x) d x}\right)^{N-1}\right] \\
& \times N y\left(x_{f i r s t}\right)\left[\int_{x_{f i r s t}}^{D} y(x) d x\right]^{N-1} d x_{f i r s t} \\
& +\int_{D-d}^{D} N y\left(x_{\text {first }}\right)\left[\int_{x_{\text {first }}}^{D} y(x) d x\right]^{N-1} d x_{\text {first }}
\end{aligned}
$$

\section{OPTIMAL DISTRIBUTION}

Our problem is to find the optimal function y which minimizes $\Lambda(y)=P_{(D, N)}$. It is a typical variational problem, and we use the classical tools of the variational calculus. Let $y_{\varepsilon}(x)=y(x)+\varepsilon h(x)$ where $h$ is any continuous function on $[0 ; D]$. If $y$ achieves the minimum of $\Lambda(y)$, then $\left(\frac{d \Lambda\left(y_{\varepsilon}\right)}{d \varepsilon}\right)_{\varepsilon=0}=0$. Then, by differentiating $\Lambda\left(y_{\varepsilon}\right)$ in order to obtain a condition on the optimal $y$ and by using the fact that the fundamental lemma of the variational calculus stands that, for any function $g$ such as $g(a)=g(b)=$ $0, \int_{a}^{b} f(u) g(u) d u=0$ implies $\forall u \in[a ; b], f(u)=0$, it can be schown that $\left(\frac{d \Lambda\left(y_{\varepsilon}\right)}{d \varepsilon}\right)_{\varepsilon=0}=0$ implies:

$$
\left\{\begin{aligned}
\forall x \in[(2 k+1) d ; 2(k+1) d], y(x)=0 \\
\forall x \in[2 k d ;(2 k+1) d], \\
\quad y(x)=y(x+2 d)\left[\frac{\int_{x+2 d}^{D} y(u) d u}{\int_{2(k+1) d}^{D} y(u) d u}\right]^{N-2}
\end{aligned}\right.
$$

$y(x)$ can thus be arbitrary chosen on the last interval of the type $[2 k d ;(2 k+1) d]$ of the $[0 ; D]$ interval as long as $\forall x \in[0 ; D], y(x) \geq 0$ and $\int_{0}^{D} y(u) d u=1$.

\section{COLLISION RATE FOR THE OPTIMAL DISTRIBUTION}

From now, without loss of generality, it is assumed $\exists n \in$ $\mathbb{N} ; D=(2 n+1) d$. Let us denote $z_{k}=\int_{2 k d}^{(2 k+1) d} y(u) d u$. Only taking into account that $x \in[(2 k+1) d ; 2(k+1) d], y(x)=0$, it can be easily shown from (7) that

$$
P(D, N)=1-N \sum_{k=0}^{\frac{D-d}{2 d}-1} z_{k}\left[\sum_{i=k+1}^{\frac{D-d}{2 d}-1} z_{i}\right]^{N-1}
$$

To inject the optimal distribution in (9) is exactly to consider in the calculation the property $\forall x \in[2 k d ;(2 k+1) d], y(x)=$ $y(x+2 d)\left[\frac{\int_{x+2 d}^{D} y(u) d u}{\int_{2(k+1) d}^{D} y(u) d u}\right]^{N-2}$, which gives by integration

$$
\begin{aligned}
z_{k} & =\int_{2 k d}^{(2 k+1) d} y(x) d x \\
& =y(x+2 d)\left[\frac{\int_{x+2 d}^{D} y(u) d u}{\int_{2(k+1) d}^{D} y(u) d u}\right]^{N-2} d x \\
& =\frac{\left(\sum_{i=k+1}^{\frac{D-d}{2 d}} z_{i}\right)^{N-1}-\left(\sum_{i=k+2}^{\frac{D-d}{2 d}} z_{i}\right)^{N-1}}{(N-1)\left(\sum_{i=k+1}^{\frac{D-d}{2 d}} z_{i}\right)^{N-2}}
\end{aligned}
$$

Finally, injecting (10) into (9) leads to

$$
P(D, N)=1-\left(1-z_{0}\right)^{N-1}
$$




\section{DISCUSSION}

The optimal distribution is defined by the recurrence equation (8). It is noteworthy to observe how the systematic method of the variational calculus leads automatically to a system avoiding frame overlaps as long as possible: emissions are only permitted each two intervals of length $d$ (cf. Fig. 1). Note that it does not mean that every frame must be sent at the beginning of such an interval. On the contrary, the emission of a frame is allowed at any time of an authorized interval. This is exactly the fundamental difference with the discrete case as studied in [6] or [4]. A particular attention must be paid to the choice of the initial distribution on the last non null interval of length $d$ : it must be well chosen in order to have the whole sum of the distribution on $[0 ; D]$ to be equal to 1 .

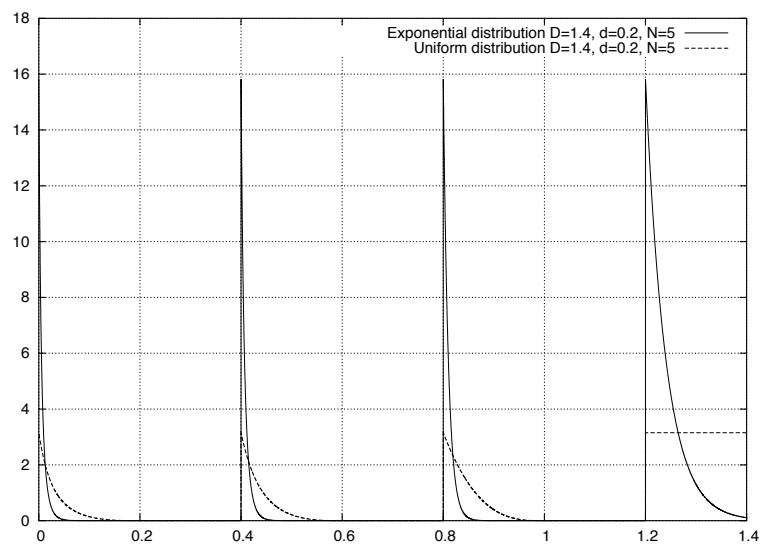

Figure 1: Examples of optimal probability density functions

In figure 1 we display some examples of the shape of the optimal distributions of the probability functions for the cases where the distribution function on the last interval of length $d$ inside the interval $[0 ; d]$ is uniform or exponential. In figure 2 several cumulative distributions functions are presented for the case where $D=1.4, d=0.2$, for different values for $N$. It can be observed that the higher $N$ is, the later the convergence of the distribution toward 1.0 is. Some

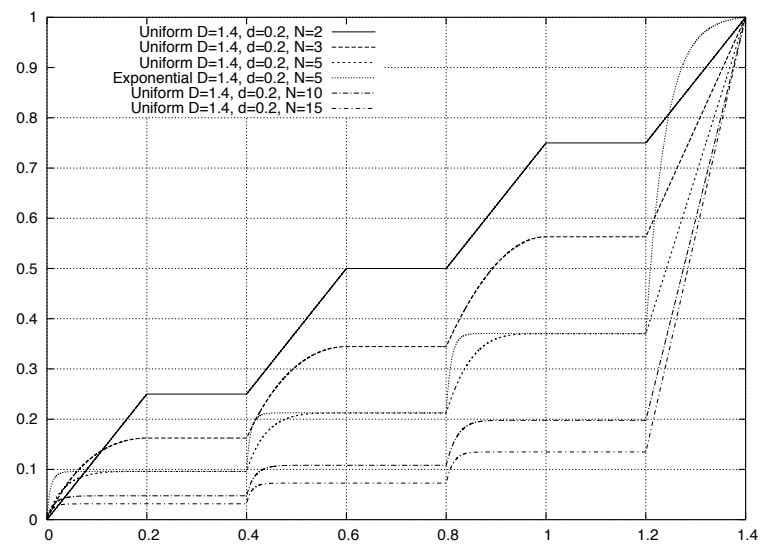

Figure 2: Examples of optimal cumulative probability functions example of collision rates are given in figure 3, together with a comparison with a simple uniform distribution.

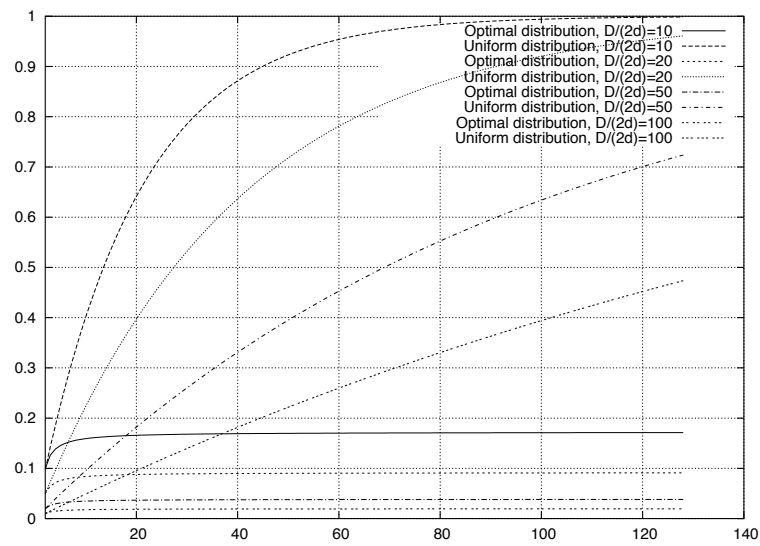

Figure 3: Examples of collision rates in function of the number of users

We are currently validating an explicit solution of the differential system (8).

\section{REFERENCES}

[1] R. Kacimi, R. Dhaou, A.-L. Beylot - Placide: An Ad Hoc Wireless Sensor Network for Cold Chain Monitoring. International Conference on Performance Modelling and Evaluation of Heterogeneous Networks (HET-NET), Karlskrona, Sweden, $18 / 02 / 2008-20 / 02 / 2008$

[2] C. Diallo, A. Gupta, M. Marot, M. Becker - Virtual base station election for wireless sensor networks. NOTERE '08: Proceedings of the 8th international conference on New technologies in distributed systems, pp. 1-7, Lyon, France, 2008.

[3] A. Delye de Clauzade de Mazieux, M. Marot, M. Becker - Correction, Generalisation and Validation of the "Max-Min d-Cluster Formation Heuristic". Networking 2007, Atlanta, GA, USA, pp.1149-1152.

[4] Y.C. Tay, K. Jamieson, H. Balakrishnan, Collision-minimizing CSMA and its applications to wireless sensor networks. Communications, IEEE Journal on , vol.22, no.6, pp. 1048-1057, Aug. 2004.

[5] T. Watteyne, I. Augé-Blum, M. Dohler, D. Barthel Reducing Collision Probability in Wireless Sensor Network Backoff-Based Election Mechanisms.. Global Telecommunications Conference, 2007. GLOBECOM '07. IEEE , vol., no., pp.673-677, 26-30 Nov. 2007.

[6] C. Bettstetter, G. Brandner, R. Vilzmann - On colliding first messages in slotted ALOHA. Personal, Indoor and Mobile Radio Communications, 2008. PIMRC 2008. IEEE 19th International Symposium on, pp.1-6, 15-18 Sept. 2008.

[7] G. Brandner, U. Schilcher, M. Gyarmati, C. Bettstetter - Non-Colliding First Messages in Slotted ALOHA: Further Insights Toward a Practical Solution. In Proc. IEEE Vehicular Technology Conf. (VTC), Barcelona, Spain, April 26-29, 2009. 